\title{
Aspartic Acid
}

National Cancer Institute

\section{Source}

National Cancer Institute. Aspartic Acid. NCI Thesaurus. Code C29608.

A non-essential amino acid in humans, Aspartic Acid has an overall negative charge and plays an important role in the synthesis of other amino acids and in the citric acid and urea cycles. Asparagine, arginine, lysine, methionine, isoleucine, and some nucleotides are synthesized from aspartic acid. Aspartic acid also serves as a neurotransmitter. ( $\mathrm{NCl04)}$ 